\title{
Iranian Nurses Perception and Practices for Delirium Assessment in Intensive Care Units
}

\author{
Fereshte Biyabanaki ${ }^{1}$, Mansour Arab², Mahlagha Dehghan ${ }^{3}$
}

\begin{abstract}
Introduction: Delirium is a fluctuating cognitive disorder that occurs in admitted patients, especially in patients who are in intensive care units. Nurses due to persistent contact with patients and direct observation of their mental changes play an essential role in delirium evaluation. Early detection of delirium, identification of risk factors, and its prevention methods are critical to reducing complications, mortality, and treatment costs. This study aimed to determine the perception and the practices of nurses in intensive care units to assess delirium and its barriers.

Study design: A cross-sectional study.

Materials and methods: All nurses working in the intensive care unit (neurology, trauma, surgery, general, and heart) of educational hospitals in Kerman, Iran, were the study population. The data gathering tool was a questionnaire consisting of four sections: demographic information, nurses' perception, practices, and perceived barriers in delirium assessment.

Results: The total score of nurses' perception in delirium assessment was $19.47 \pm 3.36$, which was higher than the medium score of the questionnaire (estimated score $=16$ ). In all, $45.5 \%$ of nurses reported having delirium treatment protocol in their units, and $12.1 \%$ of the nurses considered delirium as a priority of evaluating the patient's condition. The most important barrier to delirium assessment was the difficulty of assessing delirium in intubated patients. There was no association between nurses' perception and practices ( $p$ value $>0.05)$.

Conclusion: Designing and implementing educational programs for improving nurses' practices in this field is necessary.

Clinical significance: Healthcare providers, especially nurses, should be aware of the delirium assessment of the ICU patients to provide better care. Keywords: Assessment, Barriers, Delirium, Intensive care unit, Nurses, Perception, Practices.

Indian Journal of Critical Care Medicine (2020): 10.5005/jp-journals-10071-23502
\end{abstract}

\section{INTRODUCTION}

Delirium is a fluctuating cognitive disorder that occurs over a short period. ${ }^{1}$ According to the definition of Diagnostic and Statistical Manual of Mental Disorders (DSM-V), delirium is a disturbance of consciousness, attention, and concentration, which usually has a sudden onset and fluctuates throughout the day. ${ }^{2-4}$ Delirium is a common disorder in hospitalized patients which occurs with various clinical manifestations, including attention disorder, perception disorder, memory disorder, awareness disorder, reasoning disorder, change in cognitive function and emotional disorders, such as depression, anxiety, fear, irritability, and euphoria. Some of the factors in the development of delirium include age, mechanical ventilation, aggressive procedures, high blood pressure, alcohol consumption, metabolic disorders, uremia, hypoxia, anemia, acidosis, visual and auditory disorders, surgical interventions, and the use of drugs such as opioids and sedatives. ${ }^{5}$

The incidence of delirium in patients admitted to the intensive care unit is $32-87 \% .{ }^{6}$ There are few studies conducted in this area in Iran. In the survey of Jannati et al., the incidence of delirium after cardiac surgery was $28.5 \%{ }^{7}$ Delirium incidence increases the duration of hospitalization, ${ }^{1,2,5,8}$ the length of mechanical ventilation, $^{1,5,9} \operatorname{costs}^{1,8,10}$ the morbidity, ${ }^{2,11,12}$ the mortality, ${ }^{2,5,10-12}$ and long-term complications, including cognitive impairment and functional problems. ${ }^{2,5}$

According to the prevalence and consequences of delirium, its assessment and diagnosis in patients are essential. ${ }^{13}$ There are various tools for assessing delirium by a doctor or nurse that each of them has a different sensitivity and specificity. ${ }^{7}$ In intensive care units, Confusion Assessment Method for the ICU (CAM-ICU) and
${ }^{1}$ Critical Care Ward, Isfahan University of Medical Sciences, Isfahan, Iran ${ }^{2}$ Medical Surgical Department, Faculty of Nursing and Midwifery, Bam University of Medical Sciences, Bam, Iran

${ }^{3}$ Nursing Research Center, Critical Care Department, Nursing and Midwifery School, Kerman University of Medical Sciences, Kerman, Iran Corresponding Author: Mahlagha Dehghan, Nursing Research Center, Critical Care Department, Nursing and Midwifery School, Kerman University of Medical Sciences, Kerman, Iran, Phone: +98 3431325192, e-mail: m_dehghan@kmu.ac.ir

How to cite this article: Biyabanaki F, Arab M, Dehghan M. Iranian Nurses Perception and Practices for Delirium Assessment in Intensive Care Units. Indian J Crit Care Med 2020;24(10):955-959.

Source of support: Nil

Conflict of interest: None

Intensive Care Delirium Screening Checklist are the most widely used tools, ${ }^{14-16}$ because these two tools are easy to use for patients who cannot speak, and the patient's examination by these two tools takes place in less time and requires a brief education. ${ }^{17,18}$ Tomasi reported the sensitivity and specificity of the CAM-ICU tool $93 \%$ and $89 \%$, respectively, for detecting delirium. The ICDSC tool also has a higher sensitivity (99\%) and lower specificity (64\%) than CAM-ICU. ${ }^{19}$

Nurses in intensive care units have an important role in prevention, early diagnosis, and taking care of patients with delirium. ${ }^{2,9,10}$ Studies show that nurses do not have sufficient knowledge about delirium and their screening tools, ${ }^{1,2,20}$ and the use of standard instruments for delirium screening is not common in intensive care units, so delirium is often neglected 
and not detected. ${ }^{11,21,22}$ A study by Devlin et al. found that nurses of intensive care units had a good perception of delirium, but they give less priority to screening and dealing with this disorder. ${ }^{10}$ Glynn's study also showed that nurses' good perception did not result in their good performance. Despite the fact that the majority of respondents considered delirium as a common, serious, and undetectable syndrome, only $17.9 \%$ of them performed delirium assessment. ${ }^{23}$ In Ely's study, 92\% of the participants considered delirium to be a severe problem, but only $40 \%$ of them routinely assessed delirium in patients. ${ }^{24}$

Based on what has been mentioned, delirium and its complications in intensive care units are important, and nurses play an important role in the prevention and early diagnosis of this disorder. Considering that the previous studies in Iran on delirium assessment in nursing care are limited, the study aimed to determine the perception and the practices of nurses in intensive care units in assessing delirium and their perceived barriers.

\section{Materials and Methods}

\section{Study Design and Setting}

This was a cross-sectional study carried out on all nurses working in intensive care units of three main educational hospitals in Kerman. These hospitals are the three biggest and most advanced care centers in east south of Iran.

\section{Sample Size and Sampling}

Because of the limited number of study population, census method was used for sampling. All nurses working in the intensive care units (neurology, trauma, surgery, general, and heart) of educational hospitals in Kerman, Iran, were the study population. At the time of data collection, totally 236 nurses were screened for eligibility, i.e., having BSC or higher qualifications. From the 236 nurses, 167 who met the inclusion criteria participated in the study.

\section{Instrument}

The data collection tool was a questionnaire consisting of four parts. The questionnaire included participants demographic information, including age, gender, degree, employment status, organizational status, work experience, work experience in an intensive care unit, average hours of work in a month, the type of job shift, hospital and district service, and training course on delirium. The delirium perception, the delirium assessment practice, and the barriers sections were developed by Devlin et al. in their study in $2008 .^{10}$

The delirium assessment perception section consisted of nine items. The first eight items had a five-point Likert-type scale ranged from "I strongly agree $=4$ " to "No idea $=0$," and the last one was an open-ended question. The maximum score of this questionnaire was 32 , and the minimum score was 0 . Higher scores indicated higher delirium assessment perception.

Six items were about the delirium assessment practice. The first four items, nurses' practice in delirium evaluation in intensive care units, was compared to sedation evaluation because nurses usually do sedation assessment in intensive care units. The fifth item was about the delirium evaluating method and the frequency of its assessment in a work shift. In the sixth item, respondents were asked to compare and prioritize delirium's assessment to other critical care items such as changes in consciousness level. Each practice was described with frequency and percentage.

The delirium assessment barriers questionnaire had 13 questions and answers of the first 12 questions ranged from "I strongly agree $=4$ " to "I strongly disagree $=0$ ". The last one was an open-ended question. The maximum score of this questionnaire was 48 , and the minimum score was 0 . Higher scores indicated more barriers to delirium evaluation.

Since Devlin et al.'s questionnaire was in English, it was translated to Farsi through forward- and backward-translation method. After translation to determine the validity of the questionnaires, opinions of 10 faculty members were gathered. The content validity index $(\mathrm{CVI})$ for the perception, practice, and barriers sections were $1.00,0.91$, and 0.99 , respectively. For testing the reliability, the questionnaires were given to 10 ICU nurses with an interval of 10 days. Intraclass correlation coefficient (ICC) for the perception, practice, and barriers sections were $0.84,0.92$, and 0.88 , respectively.

\section{Data Collection}

At first, explanations were provided regarding the research process and the confidentiality of the information to the research units. All nurses were assured that their participation in the study was completely optional, and they could withdraw whenever they want mentioning that this matter will not affect their job situation in anyways. Then, informed consent was obtained from all the participants. Sampling was started after obtaining a license from the authorities of investigating units. Nurses themselves completed this questionnaire. To prevent the impact of the perception questionnaire on answering the other questionnaires, practices questionnaire was first provided to the participants. Special boxes for placing completed questionnaires in units were installed so that nurses participating in the study respond to the questionnaire in an appropriate time and put it inside the compartment which only the researcher had access to.

\section{Data Analysis}

The information was entered into SPSS version 25. Descriptive statistics (frequency, percentage, mean, and standard deviation) and inferential statistics (Pearson correlation coefficient, independent $t$ test, analysis of variance, or its nonparametric equivalent) were used to analyze the data.

\section{Ethical Considerations}

This research received the ethical code from "XXX" (1397.032 IR." $X X X " . R E C)$. The researcher explained to participants that their participation is optional and they can withdraw whenever they want. It was also assured to the nurses that the collected information was confidential and would be used only for research. Informed consent was taken from the nurses.

\section{Results}

The mean age of nurses was $31.57 \pm 6.00$. The average experience of nurses was $7.94 \pm 5.97$ years, and the average experience of nurses in intensive care unit was $5.38 \pm 4.97$ years; $77.8 \%$ of nurses were female, and $88.6 \%$ of nurses had the bachelor's degree. In all, $70.7 \%$ of participants had a history of receiving sedation information, and $62.3 \%$ had a history of receiving information about delirium which in both cases, they were often acquainted with these issues at the education time. The total score of nurses' perception of the delirium evaluation was $19.47 \pm 3.36$, which was higher than the medium questionnaire score (estimated score = 16). The highest mean was for "Delirium is an underdiagnosed problem," and the lowest was for "Delirium is associated with higher patient mortality" (Table 1). 
In all, $63.5 \%$ of nurses were aware of the existence of a protocol or clinical guidelines for sedation, and $45.5 \%$ were aware of the delirium review protocol in their service area: $87.2 \%$ of nurses, when they were taking care of patients in ICU, always or often evaluated the sedation level (Table 2); $74.5 \%$ of nurses always or often checked for delirium (Table 2); $46.4 \%$ of nurses evaluated 2-3 times the patient's ability to follow commands; and $49.1 \%$ also assessed the presence of agitation in a 6-hour shift. 38.2\% and $47.3 \%$ of nurses were not familiar with the CAM-ICU, and the ICDS checklist,

Table 1: Research units responds to perception questionnaire regarding delirium assessment

\begin{tabular}{|c|c|c|c|}
\hline Sl. no. & Item & Mean & $\begin{array}{l}\text { Standard } \\
\text { deviation }\end{array}$ \\
\hline 1 & $\begin{array}{l}\text { Delirium is an underdiagnosed } \\
\text { problem. }\end{array}$ & 2.99 & 0.69 \\
\hline 2 & $\begin{array}{l}\text { Delirium is a common response to the } \\
\text { ICU environment. }\end{array}$ & 2.77 & 0.80 \\
\hline 3 & $\begin{array}{l}\text { Delirium is a problem that requires } \\
\text { active interventions on the part of } \\
\text { caregivers.* }\end{array}$ & 2.93 & 0.83 \\
\hline 4 & $\begin{array}{l}\text { Delirium is associated with higher } \\
\text { patient mortality.* }\end{array}$ & 1.72 & 1.14 \\
\hline 5 & $\begin{array}{l}\text { ICU patients with delirium are rarely } \\
\text { agitated. }\end{array}$ & 1.93 & 0.68 \\
\hline 6 & $\begin{array}{l}\text { Initiation of antipsychotic therapy } \\
\text { (e.g., Haldol) should be the initial } \\
\text { intervention for all patients with } \\
\text { delirium. }\end{array}$ & 2.35 & 1.10 \\
\hline 7 & $\begin{array}{l}\text { Delirium is challenging to assess in } \\
\text { ICU patients. }\end{array}$ & 2.21 & 1.15 \\
\hline 8 & $\begin{array}{l}\text { Patients with delirium usually have } \\
\text { symptoms that are consistent over the } \\
\text { entire nursing shift. }\end{array}$ & 2.57 & 1.13 \\
\hline 9 & $\begin{array}{l}\text { The total score of the nurses' percep- } \\
\text { tual questionnaire regarding delirium } \\
\text { assessment }\end{array}$ & 19.47 & 3.66 \\
\hline
\end{tabular}

respectively, $24.4 \%$ and $33.3 \%$ of them never used these tools to assess delirium. Also, $69.7 \%$ of nurses rarely or never requested psychiatric counseling for their patient to evaluate delirium. 85\% of the nurses considered the altered level of consciousness, $46.1 \%$ improper placement of invasive devices, $35.9 \%$ presence of pain, $26.9 \%$ presence of agitation, and $12.1 \%$ presence of delirium the priority of evaluating the patient's condition.

There was no significant statistical correlation between the perception and practice of intensive care nurses in the delirium evaluation ( $p$ value $=0.80$ ). The total score of the barriers to the evaluation of delirium was $26.18 \pm 4.92$, which was slightly higher than the median score of the questionnaire (estimated score $=$ 24). The highest mean was for "Difficult to interpret in intubated patients," and the lowest mean was for "Nurses are not required to screen for delirium in my ICU" (Table 3).

\section{Discussion}

Results of the study showed that nurses' perception were more than mid-level. A study by Devlin et al. found that nurses of intensive care units had a good perception of delirium, but they give less priority to screening and dealing with this disorder. ${ }^{10}$ In Law and Glynn's study, nurses' perception score was higher than the average. ${ }^{12,23}$ The coherence of results of the previous studies with the present study shows that nurses' perception of delirium is high. In all, $87.2 \%$ of nurses always or often evaluated the sedation level when they were taking care of patients in ICU, and $74.5 \%$ of nurses always or often checked for delirium. Based on the findings of Devlin et al., $40 \%$ of nurses stated that they did not have delirium examination in their sedation protocol or they were not certain about it. In all, $98 \%$ of nurses assessed sedation levels in patients routinely and $47 \%$ of them always or often evaluated delirium in patients. ${ }^{10}$ Most of the nurses monitor the sedation level in patients admitted to intensive care units. However, a lower number of nurses evaluated delirium in their patients, which seems to be due to the lack of nurses' knowledge about the importance of delirium. In the present study, $38.2 \%$ and $47.3 \%$ of nurses were not familiar with the Confusion Assessment Method for the intensive care unit and the Intensive Care Delirium Screening Checklist. In the study of Elliott et al., 53\% of nurses knew CAM-ICU tools and $16 \%$ of nurses knew ICDSC tools.

Table 2: Responds to the presence of the protocol in unit, delirium evaluation and sedation level in patients admitted to the ICU

\begin{tabular}{|c|c|c|c|c|c|c|c|c|c|}
\hline \multirow[b]{2}{*}{ SIno. } & \multirow[b]{2}{*}{ Items } & \multicolumn{3}{|c|}{ Yes } & \multicolumn{3}{|c|}{ No } & \multicolumn{2}{|c|}{ Not sure } \\
\hline & & Frequency & \multicolumn{2}{|l|}{ Percent } & ency & \multicolumn{2}{|l|}{ Percent } & Frequency & Percent \\
\hline 1 & $\begin{array}{l}\text { My ICU has a sedation protocol/ } \\
\text { guideline. }\end{array}$ & 106 & \multicolumn{2}{|l|}{63.5} & 33 & \multicolumn{2}{|l|}{19.8} & 28 & 16.8 \\
\hline 2 & $\begin{array}{l}\text { Does your ICU sedation protocol } \\
\text { specify a frequency by which } \\
\text { delirium should be assessed? }\end{array}$ & 76 & \multicolumn{2}{|l|}{45.5} & & \multicolumn{2}{|l|}{41.9} & 21 & 12.6 \\
\hline \multirow[t]{4}{*}{3} & How often do you evaluate & & \multicolumn{3}{|c|}{ Always/frequently } & \multicolumn{4}{|c|}{ Rarely/never } \\
\hline & $\begin{array}{l}\text { patients for level of sedation } \\
\text { and presence of delirium? }\end{array}$ & Frequency & & Percent & & Frequency & & Percent & \\
\hline & Level of sedation* & 144 & & 87.2 & & 22 & & 13.2 & \\
\hline & Presence of delirium* & 123 & & 74.5 & & 42 & & 25.5 & \\
\hline \multirow[t]{4}{*}{4} & Indicate the frequency per every & \multicolumn{2}{|c|}{1 time } & \multicolumn{2}{|c|}{$2-3$ times } & \multicolumn{2}{|c|}{$4-6$ times } & \multicolumn{2}{|c|}{$>6$ times } \\
\hline & $\begin{array}{l}\text { 6-hour shift that you conduct } \\
\text { each evaluation }\end{array}$ & Frequency & Percent & Frequenc & Percent & Frequency & Percent & Frequency & Percent \\
\hline & Level of sedation * & 54 & 32.9 & 87 & 53.0 & 19 & 11.6 & 4 & 2.4 \\
\hline & Presence of delirium * & 61 & 38.9 & 76 & 48.4 & 16 & 9.6 & 4 & 2.4 \\
\hline
\end{tabular}

*Missing value 
Table 3: Research units respond to the questionnaire of delirium assessment barriers

\begin{tabular}{|c|c|c|c|}
\hline SI no. & Delirium screening barriers & Mean & $\begin{array}{l}\text { Standard } \\
\text { deviation }\end{array}$ \\
\hline 1 & $\begin{array}{l}\text { I do not have enough information } \\
\text { about the delirium and its evaluation. }\end{array}$ & 2.50 & 0.70 \\
\hline 2 & $\begin{array}{l}\text { Delirium assessment tools are too } \\
\text { complex to use. }\end{array}$ & 2.25 & 0.1 \\
\hline 3 & $\begin{array}{l}\text { Difficult to interpret in intubated } \\
\text { patients. }\end{array}$ & 2.93 & 0.80 \\
\hline 4 & $\begin{array}{l}\text { Do not feel confident in my ability to } \\
\text { use delirium assessment tools. }\end{array}$ & 2.17 & 1.07 \\
\hline 5 & $\begin{array}{l}\text { Do not feel that using delirium } \\
\text { assessment tool improves outcome. }\end{array}$ & 1.83 & 0.92 \\
\hline 6 & $\begin{array}{l}\text { Inability to adequately document } \\
\text { delirium assessments. }\end{array}$ & 2.31 & 0.92 \\
\hline 7 & $\begin{array}{l}\text { Inability to complete assessment in } \\
\text { the sedated patient. }\end{array}$ & 2.29 & 0.81 \\
\hline 8 & $\begin{array}{l}\text { Not enough time to perform } \\
\text { assessment (too time consuming). }\end{array}$ & 1.69 & 0.71 \\
\hline 9 & $\begin{array}{l}\text { Nurses are not required to screen for } \\
\text { delirium in my ICU. }\end{array}$ & 2.31 & 1.02 \\
\hline 10 & $\begin{array}{l}\text { In the ICU, there are more important } \\
\text { subjects than delirium, such as } \\
\text { sickness }\end{array}$ & 1.90 & 0.94 \\
\hline 11 & $\begin{array}{l}\text { Physicians already complete delirium } \\
\text { assessments. }\end{array}$ & 1.90 & 0.94 \\
\hline 12 & $\begin{array}{l}\text { Physicians do not use my assessment } \\
\text { in their decision-making. }\end{array}$ & 1.90 & 1.00 \\
\hline \multicolumn{2}{|c|}{$\begin{array}{l}\text { The total score of the Delirium Assessment } \\
\text { Obstacle Questionnaire }\end{array}$} & 26.18 & 4.92 \\
\hline
\end{tabular}

Despite the presence of delirium evaluation guidelines, nurses seem not to be familiar enough with the delirium screening tools and do not use these tools in their clinical care, and consequently, this disorder is not adequately addressed in patients admitted to intensive care units. There was no significant statistical relationship between the perception and practice of intensive care nurses in the delirium evaluation. Devlin shows that there is no difference between the perception of nurses who evaluate delirium using tools and nurses who do not carry out the assessment. ${ }^{10}$ Glynn's study also showed that nurses' good perception did not result in their good practice. Although the majority of respondents considered delirium as a common, serious, and undetectable syndrome; only $17.9 \%$ of them performed delirium assessment. ${ }^{23}$ In Ely's study, $92 \%$ of the participants considered delirium to be a serious problem, but only $40 \%$ of them routinely evaluated delirium in patients, and $16 \%$ of them used various tools to evaluate delirium. ${ }^{24}$ In Özsaban and Acaroglu study, almost all of the nurses perceived delirium as a problem and serious problem for intensive care unit patients and more than half of the nurses performed delirium assessments. However, the proportion of nurses who use delirium assessment tools was quite low. ${ }^{22}$ It is expected that a person who has a higher understanding of the subject can show a better performance, but the results of the present and mentioned studies are contrary to this. The reason for this inconsistency in the findings can be explained in such a way that, in addition to sufficient knowledge and high understanding, other factors such as the proper motivation and continuous monitoring and control system are effective to function properly. Since the appropriate provision of healthcare services is based on enough awareness and understanding, then it needs to take some steps to improve the quality-of-care services and patient satisfaction. Regarding the important role of nurses' knowledge and practice in the prevention of delirium, continuous education is needed to increase the perception and modification of health behaviors for nurses. In the barriers questionnaire, the highest mean was for "Difficult to interpret in intubated patients" and the lowest mean was for "Nurses are not required to screen for delirium in my ICU." The most important barrier to delirium evaluation in the Devlin study has mentioned the presence of a tracheal tube, the complexity of the tools, and the difficulty of evaluating patients under the sedation. ${ }^{10}$ Glynn respectively mentioned the presence of a tracheal tube, the difficulty of assessing patients under the sedation and the lack of physicians' use of nursing assessments for making decisions as these. ${ }^{23}$ In the Nydahl et al.'s study, lack of time and missing knowledge about delirium and its assessment were the most cited barriers among nurses and physicians. ${ }^{25}$ The result of present study is consistent with the three studies mentioned above, and the presence of the endotracheal tube was one of the most important barriers to evaluate the delirium. CAM-ICU and ICDSC are specifically used for patients with intubation and admission to intensive care units. Nurses do not seem to have enough knowledge about the delirium screening tools.

This study has some limitations. We used a long and selfadministered questionnaire to examine the nurses' perception, practices, and their perceived barriers for delirium assessment which may affect on precise responses of the nurses. However, our response rate was high. Also, we used convenience sampling so the results should generalize to other communities with cautious.

\section{ConClusion}

It is concluded from the findings of this study that the research units do not have enough perception to evaluate delirium and its diagnostic tools, and despite the presence of guidelines for delirium evaluation, nurses are not familiar enough with the delirium screening tools and do not use these tools in their clinical care, and consequently, this disorder is not adequately evaluated in patients admitted to intensive care units.

\section{Clinical Significance}

Delirium is a common disorder in hospitalized patients, especially in patients admitted to intensive care units. Nurses in intensive care units have an important role in prevention, early diagnosis, and taking care of patients with delirium. The majority of the nurses in intensive care units assessed patients for sedation level and delirium; however, they did not check the patients according standard tools. The practice of nurses in intensive care units for delirium assessment is not optimal. Health care providers, especially nurses, should be aware of the delirium assessment of the ICU patients to provide better care. Therefore, it is recommended that these topics be included in the healthcare provider's curriculum as well as nurses with greater academic ability, higher perception, and more top skills be applied in intensive care units.

\section{Acknowledgments}

The present article has been derived from the MS thesis for intensive care nursing. We would thank the nursing staff of the intensive care units of educational hospitals of Kerman University of Medical Sciences. Many thanks for late Dr Masoud Rayyani for his coorperation. 


\section{Availability of Data and Materials}

The data sets used and/or analyzed during the current study are available from the corresponding author on reasonable request.

\section{Author Contributions}

Fereshte Biyabanaki, Mansour Arab, and Mahlagha Dehghan develop the study idea and protocol. Mansour Arab and Mahlagha Dehghan supervised the study sampling. Fereshte Biyabanaki did the sampling. Mahlagha Dehghan analyzed the data. Fereshte Biyabanaki wrote the first draft of the manuscript. All authors read and confirmed the final version of the manuscript.

\section{References}

1. Elliott SR. ICU delirium: a survey into nursing and medical staff knowledge of current practices and perceived barriers towards ICU delirium in the intensive care unit. Intensive Crit Care Nursing 2014;30(6):333-338. DOI: 10.1016/j.iccn.2014.06.004.

2. Demir Korkmaz F, Gok F, Yavuz Karamanoglu A. Cardiovascular surgery nurses' level of knowledge regarding delirium. Nurs Crit Care 2015;21(5):279-286. DOI: 10.1111/nicc.12184

3. Flaigle MC, Ascenzi J, Kudchadkar SR. Identifying barriers to delirium screening and prevention in the pediatric ICU: evaluation of PICU staff knowledge. J Pediatr Nurs 2016;31(1):81-84. DOI: 10.1016/ j.pedn.2015.07.009.

4. Middle B, Miklancie M. Strategies to improve nurse knowledge of delirium: a call to the adult-gerontology clinical nurse specialist. Clin Nur Special 2015;29(4):218-229. DOI: 10.1097/ NUR.0000000000000138.

5. Ribeiro SCL, Nascimento ERPD, Lazzari DD, Jung W, Boes AA, Bertoncello KC. Knowledge of nurses about delirium in critical patients: collective subject discourse. Texto Cont-Enfermag 2015;24(2):513-520. DOI: 10.1590/0104-07072015001702014.

6. Piao J, Jin Y, Lee SM. Triggers and Nursing influences on delirium in intensive care units. Nurs Crit Care 2018;23(1):8-15. DOI: 10.1111/ nicc. 12250

7. Jannati Y, Bagheri M, Sohrabi M, Yazdani J, Mazdarani S. Incidence of delirium and associated factors before open heart surgery. J Res Develop Nurs Midwif 2013;10(1):33-42.

8. Trogrlić Z, Ista E, Ponssen HH, Schoonderbeek JF, Schreiner $F$, Verbrugge $\mathrm{SJ}$, et al. Attitudes, knowledge and practices concerning delirium: a survey among intensive care unit professionals. Nurs Crit Care 2017;22(3):133-140. DOI: 10.1111/nicc.12239.

9. Zamoscik K, Godbold R, Freeman P. Intensive care nurses' experiences and perceptions of delirium and delirium care. Intensive Crit Care Nursing 2017;40:94-100. DOI: 10.1016/j.iccn.2017.01.003.

10. Devlin JW, Fong JJ, Howard EP, Skrobik Y, McCoy N, Yasuda C, et al. Assessment of delirium in the intensive care unit: nursing practices and perceptions. Am J Crit Care 2008;17(6):555-565. DOI: 10.4037/ ajcc2008.17.6.555.

11. Kang Y, Moyle W, Cooke $M, O^{\prime} D$ wyer ST. An educational programme to improve acute care nurses' knowledge, attitudes and family caregiver involvement in care of people with cognitive impairment. Scand J Caring Sci 2016;31(3):631-640. DOI: 10.1111/scs.12377.

12. Law TJ, Leistikow NA, Hoofring L, Krumm S, Neufeld K, Needham D. A survey of nurses' perceptions of the intensive care delirium screening checklist. Dynamics 2012;23(4):18-24.

13. Jung J-H, Lim J-H, Kim E-J, An H-C, Kang M-K, Lee J, et al. The experience of delirium care and clinical feasibility of the CAM-ICU in a Korean ICU. Clin Nurs Res 2013;22(1):95-111. DOI: 10.1177/1054773812447187.

14. Nishimura K, Yokoyama K, Yamauchi N, Koizumi M, Harasawa N, Yasuda T, et al. Sensitivity and specificity of the confusion assessment method for the intensive care unit (CAM-ICU) and the intensive care delirium screening checklist (ICDSC) for detecting post-cardiac surgery delirium: a single-center study in Japan. Heart Lung 2016;45(1):15-20. DOI: 10.1016/j.hrtlng.2015.11.001.

15. Luetz A, Heymann A, Radtke FM, Chenitir C, Neuhaus U, Nachtigall I, et al. Different assessment tools for intensive care unit delirium: which score to use? Crit Care Med 2010;38(2):409-418. DOI: 10.1097/ CCM.0b013e3181cabb42.

16. Svenningsen $\mathrm{H}$. A shared language regarding sedation and delirium in critically ill patients. Nurs Crit Care 2015;20(4):204-209. DOI: 10.1111/ nicc.12187.

17. Scott P, Mcllveney F, Mallice M. Implementation of a validated delirium assessment tool in critically ill adults. Intensive Crit Care Nursing 2013;29(2):96-102. DOI: 10.1016/j.iccn.2012.09.001.

18. van Eijk MMJ, van Marum RJ, Klijn IAM, de Wit N, Kesecioglu J, Slooter AJC. Comparison of delirium assessment tools in a mixed intensive care unit. Crit Care Med 2009;37(6):1881-1885. DOI: 10.1097/ CCM.0b013e3181a00118.

19. Tomasi CD, Grandi C, Salluh J, Soares M, Giombelli VR, Cascaes S, et al. Comparison of CAM-ICU and ICDSC for the detection of delirium in critically ill patients focusing on relevant clinical outcomes. J Crit Care 2012;27(2):212-217. DOI: 10.1016/j.jcrc.2011.05.015.

20. van de Steeg $L$, IJkema $R$, Wagner $C$, Langelaan $M$. The effect of an e-learning course on nursing staff's knowledge of delirium: a before-and-after study. BMC Med Educ 2015;15(1):1. DOI: 10.1186/ s12909-015-0289-2.

21. Rowley-Conwy G. Barriers to delirium assessment in the intensive care unit: A literature review. Intensive Crit Care Nurs 2018;44:99-104. DOI: 10.1016/j.iccn.2017.09.001.

22. Özsaban A, Acaroglu R. Delirium assessment in intensive care units: practices and perceptions of Turkish nurses. Nurs Crit Care 2016;21(5):271-278. DOI: 10.1111/nicc.12127.

23. Glynn L, Corry M. Intensive care nurses' opinions and current practice in relation to delirium in the intensive care setting. Intensive Crit Care Nurs 2015;31(5):269-275. DOI: 10.1016/j.iccn.2015.05.001.

24. Ely EW, Stephens RK, Jackson JC, Thomason JWW, Truman B, Gordon $\mathrm{S}$, et al. Current opinions regarding the importance, diagnosis, and management of delirium in the intensive care unit: a survey of 912 healthcare professionals. Crit Care Med 2004;32(1):106-112. DOI: 10.1097/01.CCM.0000098033.94737.84.

25. Nydahl P, Dewes M, Dubb R, Hermes C, Kaltwasser A, Krotsetis $S$, et al. Survey among critical care nurses and physicians about delirium management. Nurs Crit Care 2018;23(1):23-29. DOI: 10.1111/ nicc.12299. 